TRANSACTIONS OF THE

AMERICAN MATHEMATICAL SOCIETY

Volume 359, Number 8, August 2007, Pages 3529-3547

S 0002-9947(07)04417-0

Article electronically published on March 20, 2007

\title{
ON OPERATOR-VALUED FOURIER MULTIPLIER THEOREMS
}

\author{
ŽELJKO ŠTRKALJ AND LUTZ WEIS
}

\begin{abstract}
The classical Fourier multiplier theorems of Marcinkiewicz and Mikhlin are extended to vector-valued functions and operator-valued multiplier functions on $\mathbb{Z}^{d}$ or $\mathbb{R}^{d}$ which satisfy certain $R$-boundedness conditions.
\end{abstract}

\section{INTRODUCTION AND MOTIVATION}

Let $X$ and $Y$ be real (or complex) Banach spaces and $\mathscr{B}(X, Y)$ the Banach space of bounded linear operators $T: X \rightarrow Y$, endowed with the usual operator norm. By $\mathscr{S}\left(\mathbb{R}^{d} ; X\right)$ we denote the Schwartz space of rapidly decreasing functions from $\mathbb{R}^{d}$ to $X$ and by $\wedge, \vee$ we denote the Fourier transform and the inverse Fourier transform. For $1 \leq p<\infty$ let $L^{p}\left(\mathbb{T}^{d} ; X\right)$ and $L^{p}\left(\mathbb{R}^{d} ; X\right)$ be the usual Bochner spaces of $p$-integrable $X$-valued functions on the $d$-dimensional circle $\mathbb{T}^{d}$ and $\mathbb{R}^{d}$ respectively.

In the first part of this article we are interested in obtaining Fourier multiplier theorems on $L^{p}\left(\mathbb{T}^{d} ; X\right)$ in the following sense.

For $z \in \mathbb{T}^{d}$ set $\mathfrak{e}_{\mathfrak{x}}(z)=z^{\mathfrak{x}}, \mathfrak{x} \in \mathbb{Z}^{d}$. We say that a function $M: \mathbb{Z}^{d} \rightarrow \mathscr{B}(X, Y)$ is a Fourier multiplier on $L^{p}\left(\mathbb{T}^{d} ; X\right)$ if the operator

$$
f=\sum_{\mathfrak{x} \in \mathbb{Z}^{d}} \hat{f}(\mathfrak{x}) \otimes \mathfrak{e}_{\mathfrak{x}} \longmapsto \mathscr{K}_{M} f=\sum_{\mathfrak{x} \in \mathbb{Z}^{d}} M(\mathfrak{x}) \hat{f}(\mathfrak{x}) \otimes \mathfrak{e}_{\mathfrak{x}},
$$

first defined for $f$ with a finitely valued Fourier transformation $\hat{f}$, extends uniquely to a bounded operator from $L^{p}\left(\mathbb{T}^{d} ; X\right)$ to $L^{p}\left(\mathbb{T}^{d} ; Y\right)$. We denote the set of such multipliers by $\mathscr{M}_{p}\left(\mathbb{Z}^{d} ; X, Y\right)$.

For $d=1$ the assumption of the Marcinkiewicz theorem requires that for the dyadic decomposition $I_{n}=\left\{\mathfrak{x} \in \mathbb{Z}: 2^{n-1}<|\mathfrak{x}| \leq 2^{n}\right\}$ we have

$$
\operatorname{var}\left(M_{I_{n}}\right) \leq C
$$

for all $n \in \mathbb{N}$. For multipliers $M(\mathfrak{x})=m(\mathfrak{x}) I_{X}$ with a scalar function $m$ it was shown in Bou2] that (1.2) implies $M \in \mathscr{M}_{p}(\mathbb{Z} ; X)$ if and only if $X$ is a UMD-space. A UMD-space can be characterized by the fact that the special multiplier $m_{0}(\mathfrak{x})=$ $\operatorname{sign}(\mathfrak{x})$ belongs to $\mathscr{M}_{p}(\mathbb{Z} ; X)$ (see Bou1, $[\mathrm{Bu}$ ). Indeed, Bourgain shows how "to built up" general scalar multipliers with (1.2) from modifications of $m_{0}$. It is well known, that all subspaces and quotient spaces of $L^{q}(\Omega)$-spaces with $1<q<\infty$ are UMD-spaces.

For operator-valued multipliers $M(\mathfrak{x}) \in \mathscr{B}(X)$ the variation (1.2), taken with respect to the operator norm, always implies that $M \in \mathscr{M}_{p}(\mathbb{Z} ; X)$ if and only if

Received by the editors October 1, 1999 and, in revised form, April 10, 2003.

2000 Mathematics Subject Classification. Primary 42B15, 42A45, 46E40; Secondary 46B09.

(C)2007 American Mathematical Society 
$X$ is a Hilbert space (Schwartz showed that (1.2) is sufficient in Hilbert spaces $\mathrm{BL}$, and Pisier observed the converse). So besides the UMD property for $X$ and $Y$ one needs additional assumptions on the multiplier function $M$. Recently it was shown in $\mathrm{We}$, in the context of the Mikhlin-multiplier theorem for operatorvalued multipliers, that this additional condition can be expressed in terms of $R$ boundedness:

A subset $\mathcal{T} \subset \mathscr{B}(X, Y)$ is called $R$-bounded if there is a constant $C$ such that for all $T_{0}, T_{1}, \ldots, T_{n} \in \mathcal{T}, x_{0}, x_{1}, \ldots, x_{n} \in X$ and $n \in \mathbb{N}$

$$
\int_{0}^{1}\left\|\sum_{k=0}^{n} \varepsilon_{k}(t) T_{k} x_{k}\right\|_{Y} d t \leq C \int_{0}^{1}\left\|\sum_{k=0}^{n} \varepsilon_{k}(t) x_{k}\right\|_{X} d t
$$

where $\left(\varepsilon_{k}\right)$ is the sequence of Rademacher functions on $[0,1]$. This concept was already used in Bou2 and $[\mathrm{BG}$ in connection with multiplier theorems, and more recently a detailed study was given in [CPSW]. If $X=Y=L^{q}(\Omega)$ for some $1 \leq q<\infty$, then (1.3) is equivalent to the square function estimate

$$
\left\|\left(\sum_{k=0}^{n}\left|T_{k} x_{k}\right|^{2}\right)^{1 / 2}\right\|_{L^{q}} \leq \tilde{C}\left\|\left(\sum_{k=0}^{n}\left|x_{k}\right|^{2}\right)^{1 / 2}\right\|_{L^{q}}
$$

known from harmonic analysis.

In this paper we use $R$-boundedness to give an appropriate form of the Marcinkiewicz condition for operator-valued multipliers. In place of (1.2) we assume that for some absolutely convex $R$-bounded set $\mathcal{T}$ we have

$$
\sum_{k \in I_{n}}\|M(k+1)-M(k)\|_{\mathcal{T}} \leq C
$$

for all $n \in \mathbb{N}$, where $\|\cdot\|_{\mathcal{T}}$ denotes the Minkowski functional of $\mathcal{T}$. If $X$ and $Y$ are UMD-spaces, $M$ satisfies (1.5) and

$$
\left\{M\left( \pm 2^{k-1}\right) k \in \mathbb{N}\right\} \text { is } R \text {-bounded, }
$$

then we show in section 3 that $M \in \mathscr{M}_{p}(\mathbb{Z} ; X, Y)$. We also give $d$-dimensional versions of this result. Our proof follows the techniques of $\mathrm{Zi}$, who proved $d$ dimensional generalizations of Bourgain's result for scalar multipliers. In Remark 1.1 below we point out that (1.6) is necessary for $M$ to be in $\mathscr{M}_{p}(\mathbb{Z} ; X, Y)$. If $M$ belongs to $\mathscr{M}_{p}(\mathbb{Z} ; X, Y)$ and (1.2) holds, it follows that $\{M(\mathfrak{x}) \mathfrak{x} \in \mathbb{Z}\}$ is $R$-bounded, and this indicates that $R$-boundedness arises naturally in the context of multiplier theorems. The second part of the paper will treat the continuous case. In analogy to the discrete setting we say that a function $M: \mathbb{R}^{d} \backslash\{0\} \rightarrow \mathscr{B}(X, Y)$ is a Fourier multiplier, i.e. $M \in \mathscr{M}_{p}\left(\mathbb{R}^{d} ; X, Y\right)$, if the operator

$$
f \longmapsto \mathscr{K}_{M} f=(M(\cdot) \hat{f}(\cdot))^{\vee},
$$

first defined for $f \in \mathscr{S}\left(\mathbb{R}^{d} ; X\right)$, extends to a bounded operator from $L^{p}\left(\mathbb{R}^{d} ; X\right)$ to $L^{p}\left(\mathbb{R}^{d} ; Y\right)$.

For multiplier theorems of the Mikhlin type, one considers the sets

$$
\left\{|x|^{|\gamma|}\left(D^{\gamma} M\right)(x): x \in \mathbb{R}^{d} \backslash\{0\}, \gamma \leq(1, \ldots, 1)\right\}
$$

or

$$
\left\{x^{\gamma}\left(D^{\gamma} M\right)(x): x \in \mathbb{R}^{d} \backslash\{0\}, \gamma \leq(1, \ldots, 1)\right\} .
$$


Again the norm boundedness of these sets is only sufficient in Hilbert spaces. If $X$ and $Y$ are UMD-spaces and (1.8) is $R$-bounded, we show then in Theorem 4.4 that $M \in \mathscr{M}_{p}\left(\mathbb{R}^{d} ; X, Y\right)$ holds. For the finer condition (1.9) we need besides the $R$-boundedness of (1.9) and the UMD property an additional assumption on $X$ and $Y$, which is the property $(\alpha)$, introduced by Pisier (see Pi2]). In particular every $q$-concave Banach-lattice with $q<\infty$, or more generally, every Banach space with local unconditional structure and finite cotype has property $(\alpha)$ (cf. Pi2, [DJT, Theorem 14.1). We reduce these theorems to the discrete case in section 3, again following the method in [Zi].

We also give a new criterion for the $R$-boundedness of a function $x \in I \rightarrow M(x)$. For one-dimensional intervals, $R$-boundedness follows if $M$ is of bounded variation (see Theorem 2.7). For $d$-dimensional intervals we give an integrability criterion (see Theorem 4.1).

The next remark illustrates how the notion of $R$-boundedness is necessary if one considers operator-valued Fourier multipliers on vector-valued $L^{p}$-spaces.

1.1. Remark. Let us assume that we have a multiplier $M \in \mathscr{M}_{p}(\mathbb{Z} ; X, Y)$. For trigonometric polynomials $f=\sum_{k=1}^{n} x_{k} \otimes \mathfrak{e}_{2^{k}}$ we therefore obtain

$$
\left\|\sum_{k=1}^{n} M\left(2^{k}\right) x_{k} \otimes \mathfrak{e}_{2^{k}}\right\|_{L^{p}(\mathbb{T} ; Y)} \leq C_{1}\left\|\sum_{k=1}^{n} x_{k} \otimes \mathfrak{e}_{2^{k}}\right\|_{L^{p}(\mathbb{T} ; X)} .
$$

Now, it is known (see [Pi1]) that there is a universal constant $C>0$ such that for any Banach space $E$ and any finite sequence $y_{1}, \ldots, y_{n}$ in $E$ :

$$
\frac{1}{C}\left\|\sum_{k=1}^{n} \varepsilon_{k} y_{k}\right\|_{L^{p}([0,1] ; E)} \leq\left\|\sum_{k=1}^{n} y_{k} \otimes \mathfrak{e}_{2^{k}}\right\|_{L^{p}(\mathbb{T} ; E)} \leq C\left\|\sum_{k=1}^{n} \varepsilon_{k} y_{k}\right\|_{L^{p}([0,1] ; E)},
$$

where $\left(\varepsilon_{k}\right)_{k \geq 1}$ denotes the sequence of Rademacher functions on $[0,1]$. These inequalities in connection with (1.10) lead to

$$
\left\|\sum_{k=1}^{n} \varepsilon_{k} M\left(2^{k}\right) x_{k}\right\|_{L^{p}([0,1] ; Y)} \leq C_{2}\left\|\sum_{k=1}^{n} \varepsilon_{k} x_{k}\right\|_{L^{p}([0,1] ; X)} .
$$

This means that the collection $\left\{M\left(2^{k}\right): k \in \mathbb{N}\right\} \subset \mathscr{B}(X, Y)$ is $R$-bounded. By an application of Proposition 1.3 in [B] we get that $\mathcal{T}=\{M(r): r \in \mathbb{Z}\}$ is $R$-bounded.

\section{2. $R$-BOUNDEDNESS}

In this section we list some important results about $R$-bounded collections $\mathcal{T}$ of bounded linear operators. Let $(\Omega, \mathfrak{A}, P)$ be a probability space and $\left(\varepsilon_{k}\right)_{k=0}^{\infty}$ a sequence of independent symmetric $\{-1,1\}$-valued random variables on $(\Omega, \mathfrak{A}, P)$. With $L^{p}(\Omega ; X)$ we denote the Bochner space of $p$-integrable $X$-valued functions on $(\Omega, \mathfrak{A}, P)$.

2.1. Definition. A collection $\mathcal{T} \subset \mathscr{B}(X, Y)$ is said to be $\mathbf{R}$-bounded if there exist a constant $C>0$ such that for all $T_{0}, T_{1}, \ldots, T_{n} \in \mathcal{T}, x_{0}, x_{1}, \ldots, x_{n} \in X$ and all $n \in \mathbb{N}$

$$
\left\|\sum_{k=0}^{n} \varepsilon_{k} T_{k} x_{k}\right\|_{L^{1}(\Omega ; Y)} \leq C\left\|\sum_{k=0}^{n} \varepsilon_{k} x_{k}\right\|_{L^{1}(\Omega ; X)} .
$$

The smallest constant $C$, for which (2.1) holds is denoted by $R(\mathcal{T})$. 
The notion of $R$-boundedness was already implicitly used in Bou1, Bou2] and was introduced in the paper [BG]. Detailed studies about collections of $R$-bounded operators can be found in [CPSW] and [We. In We the reader will find as well a new characterization of maximal $L^{p}$-regularity of abstract differential equations using this notation.

Note. We want to emphasize that the definition of collections of $R$-bounded operators does not depend on the probability space $(\Omega, \mathfrak{A}, P)$ and the sequence of random variables $\left(\varepsilon_{k}\right)$.

2.2. Remark. Here are some rather known facts about $R$-boundedness:

(a) Using Kahane's inequality (see [LT] we can replace (2.1) by $(1 \leq p<\infty)$

$$
\left\|\sum_{k=0}^{n} \varepsilon_{k} T_{k} x_{k}\right\|_{L^{p}(\Omega ; Y)} \leq C_{p}\left\|\sum_{k=0}^{n} \varepsilon_{k} x_{k}\right\|_{L^{p}(\Omega ; X)} .
$$

(b) It is easy to see that $R$-bounded collections $\mathcal{T} \subset \mathscr{B}(X, Y)$ are necessarily bounded in $\mathscr{B}(X, Y)$. If $X$ and $Y$ are both Hilbert spaces, (2.2) shows that the converse also holds.

(c) If $X$ and $Y$ are $q$-concave Banach lattices $(1 \leq q<\infty)$ (for the Definition see [LT] 1.d.3.(iii)) the definitions (2.1) and (2.2) are equivalent to (see [LT] Theorem 1.d.6.(i))

$$
\left\|\left(\sum_{k=0}^{n}\left|T_{k} x_{k}\right|^{2}\right)^{1 / 2}\right\|_{Y} \leq C\left\|\left(\sum_{k=0}^{n}\left|x_{k}\right|^{2}\right)^{1 / 2}\right\|_{X} .
$$

(d) If $\mathcal{T}:=\left\{a_{k} I_{X}: k \in \mathbb{N}_{0}\right\} \subset \mathscr{B}(X)$, then $R(\mathcal{T}) \leq 2\|a\|_{\infty}$.

In the following we want to present four practical methods to enlarge an $R$ bounded collection $\mathcal{T}$. By $\operatorname{aco}(\mathcal{T})$ we denote the real or complex absolute convex hull of a collection $\mathcal{T} \subset \mathscr{B}(X, Y)$. With this in mind, we can formulate the first statement.

2.3. Lemma. Let $\mathcal{T} \subset \mathscr{B}(X, Y)$ be an $R$-bounded collection with $R$-bound $R(\mathcal{T})$. Then the absolute convex hull $\operatorname{aco}(\mathcal{T})$ as well as the strong closure of $\mathcal{T}$ are $R$ bounded with $R$-bounds not larger than $2 R(\mathcal{T})$.

The statement is based on ideas introduced in [Bou2. For the proof we refer to CPSW]. The next two lemmas can also be found in [CPSW].

2.4. Lemma. Let $\mathcal{S} \subset \mathscr{B}\left(X_{2}, X_{3}\right), \mathcal{T} \subset \mathscr{B}\left(X_{1}, X_{2}\right)$ be two collections which are $R$-bounded. Then the collection

$$
\mathcal{S T}=\{S T: S \in \mathcal{S}, T \in \mathcal{T}\}
$$

is $R$-bounded with an $R$-bound not greater than $R(\mathcal{S}) R(\mathcal{T})$.

Let $E$ be a Banach space, $X=L^{p}(\Lambda ; E)$ for some $\sigma$-finite measure space $(\Lambda, \mathfrak{B}, \mu)$ and $1 \leq p<\infty$. For $\varphi \in L^{\infty}(\Lambda)$ we denote by $M_{\varphi}$ the pointwise multiplication operator on $X$.

2.5. Lemma. Let $X=L^{p}(\Lambda ; E)$ and $\mathcal{T} \subset \mathscr{B}(X)$. If $\mathcal{T}$ is $R$-bounded, then the collection

$$
\left\{M_{\varphi} T M_{\psi}: \varphi, \psi \in L^{\infty}(\Lambda) \text { with }\|\varphi\|_{\infty},\|\psi\|_{\infty} \leq 1, T \in \mathcal{T}\right\}
$$

is R-bounded as well. 
The following extension result is also useful and is taken from We], Proposition 2.11 .

2.6. Lemma. For $T \in \mathscr{B}(X, Y)$ define the operator $(\tilde{T} f)(\lambda):=T(f(\lambda)), f \in$ $L^{p}(\Lambda ; X), \lambda \in \Lambda, 1 \leq p<\infty$. Then, if $\mathcal{T} \subset \mathscr{B}(X, Y)$ is $R$-bounded, the collection $\tilde{\mathcal{T}}=\{\tilde{T}: T \in \mathcal{T}\} \subset \mathscr{B}\left(L^{p}(\Lambda ; X), L^{p}(\Lambda ; Y)\right)$ is also R-bounded.

In the next theorem we give a sufficient condition on the regularity of operatorvalued functions which ensures $R$-boundednes for their range collection. This result generalizes in particular Proposition 2.5 in [We. Other statements of this type can be found in Corollary 3.5 and Theorem 4.1.

2.7. Theorem. If $X, Y$ are arbitrary Banach spaces and the function $M: I \rightarrow$ $\mathscr{B}(X, Y)$ on an interval $I=[a, b) \subset \mathbb{R}$ is of bounded variation, then the collection

$$
\mathcal{M}:=\{M(x): x \in I\}
$$

is $R$-bounded with $R(\mathcal{M}) \leq C(\|M(a)\|+\operatorname{var}(M))$.

Proof. Assume that $M$ has the form

$$
M(t)=M(a)+\sum_{j=1}^{m} \chi_{A_{j}}(t) M_{j}
$$

with $A_{j} \subset I$ and $M_{j} \in \mathscr{B}(X, Y)$. Then, using Lemma 2.4 in [We, we obtain

$$
R(\{M(x): x \in I\}) \leq\|M(a)\|+\sum_{j=1}^{m}\left\|M_{j}\right\| .
$$

We will now show that for general $M$ there is sequence of the form

$$
M_{k}(t)=M(a)+\sum_{j} \chi_{A_{k, j}}(t) M_{k, j}
$$

with

$$
\sum_{j}\left\|M_{k, j}\right\| \leq C \operatorname{var}(M) \quad \forall k \in \mathbb{N}
$$

and

$$
\left\|M_{k}(t)-M(t)\right\| \rightarrow 0
$$

for $k \rightarrow \infty$ and all $t \in I$. With this approximation property for $M$ the claim follows by an application of Lemma 2.3 .

Without loss of generality we assume that $M$ is continuous from the left. If we put $\Delta(t):=\lim _{s \backslash t} M(s)-M(t)$, the bounded variation property on $M$ states that for each $n \in \mathbb{N}$ the set

$$
\mathscr{T}_{n}:=\left\{t \in I: \frac{1}{n+1} \leq\|\Delta(t)\|<\frac{1}{n}\right\}
$$

has to be finite, i.e. $\mathscr{T}_{n}=\left\{t_{n, 1}, \ldots, t_{n, k(n)}\right\}$. If we put

$$
N(t):=\sum_{n=1}^{\infty} \sum_{x \in \mathscr{T}_{n}} \Delta(x) \chi_{(x, b)}(t),
$$

one can show that $N$ is continuous from the left and $\operatorname{var}(N) \leq \operatorname{var}(M)$ holds. Moreover the function $L:=M-N$ is also of bounded variation with $\operatorname{var}(L) \leq$ 
$2 \operatorname{var}(M)$, continuous and therefore uniformly continuous on $[a, b)$. Obviously the definition of $N$ allows us to construct a sequence $\left(N_{k}\right)$ of functions of the form

$$
N_{k}=M(a)+\sum_{j=1}^{m_{k}} \chi_{A_{k, j}}(t) N_{k, j}
$$

with $\left\|N_{k}(t)-N(t)\right\| \rightarrow 0$ for $k \rightarrow \infty$ and all $t \in[a, b)$ and $\operatorname{var}\left(N_{k}\right) \leq \operatorname{var}(N)$. To approximate $L$ we choose for a given $\varepsilon>0$ a $\delta>0$ such that $|s-t|<\delta$ implies $\|L(s)-L(t)\| \leq \varepsilon$ and that for a partition $\left(t_{j}\right)$ in $(a, b)$ with $\sup _{j}\left|t_{j}-t_{j-1}\right| \leq \delta$ we have

$$
\left|\sum_{j}\left\|L\left(t_{j}^{\prime}\right)-L\left(t_{j-1}^{\prime}\right)\right\|-\operatorname{var}(L)\right| \leq \varepsilon
$$

for all refinements $\left(t_{j}^{\prime}\right)$ of $\left(t_{j}\right)$. Now the function

$$
\tilde{L}(t):=M(a)+\sum_{j}\left[L\left(t_{j}\right)-L\left(t_{j-1}\right)\right] \chi_{\left[t_{j}, b\right)}(t)
$$

satisfies $\operatorname{var}(\tilde{L}) \leq \operatorname{var}(L)+\varepsilon$ and $\|L(t)-\tilde{L}(t)\| \leq \varepsilon$. In this way we can find a sequence $\left(L_{k}\right)$ of the form

$$
L_{k}=M(a)+\sum_{j=1}^{m_{k}} \chi_{A_{k, j}}(t) M_{j}
$$

satisfying $\left\|L_{k}(t)-L(t)\right\| \rightarrow 0$ for $k \rightarrow \infty, t \in[a, b)$ and $\operatorname{var}\left(L_{k}\right) \leq 2 \operatorname{var}(L)$. Now the sequence $M_{k}:=N_{k}+L_{k}$ has the required properties (2.5) and (2.6).

Since outside the Hilbert space setting bounded sets of operators are usually not $R$-bounded anymore, we have to replace the operator norm in various estimates and definitions by the following norms "measuring" $R$-boundedness:

2.8. Notation. For a bounded collection $\mathcal{T} \subset \mathscr{B}(X, Y)$ we denote the Minkowski functional of aco $(\mathcal{T})$ by

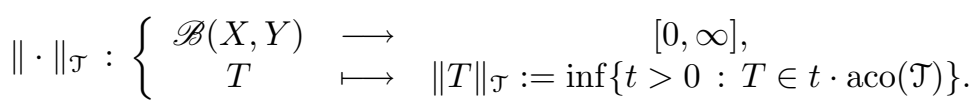

Here are some obvious facts that will be used constantly in the next two sections.

2.9. Remark. a) If we set $\mathcal{T}:=\left\{a_{k} I_{X}: k \in \mathbb{N}_{0}\right\} \subset \mathscr{B}(X)$, then we have

$$
\operatorname{aco}(\mathcal{T})=\left\{z I_{X}:|z| \leq\|a\|_{\infty}\right\}
$$

and thus by Remark 2.2.(d) we get

$$
R(\mathcal{T})\left\|\alpha I_{X}\right\|_{\mathcal{T}} \leq 2\|a\|_{\infty}\left\|\alpha I_{X}\right\|_{\mathcal{T}} \leq 2|\alpha|, \quad \alpha \in \mathbb{C} .
$$

b) Let $\mathcal{T} \subset \mathscr{B}(X, Y)$ be an $R$-bounded collection. Then the following holds:

(i) $\left\|T_{1}+T_{2}\right\|_{\mathcal{T}} \leq\left\|T_{1}\right\|_{\mathcal{T}}+\left\|T_{2}\right\|_{\mathcal{T}}$.

(ii) If a collection $\mathcal{M}=\left\{M_{n}: n \in \mathbb{N}\right\}$ has the property that

$$
C:=\sup \left\{\left\|M_{n}\right\|_{\mathcal{T}}: n \in \mathbb{N}\right\}<\infty,
$$

then $\mathcal{N}$ is also $R$-bounded and the $R$-bound is not greater than $4 C R(\mathcal{T})$. 


\section{The DisCRETE CASE}

In this section we are interested in giving sufficient conditions on the function $M: \mathbb{Z}^{d} \rightarrow \mathscr{B}(X, Y)$ so that the operator, defined in (1.1), extends to a bounded operator. In particular we want to generalize the results given in [Bou2] and [Zi].

The next two sections will rather follow the examinations given in section 1 and 2 of $\mathrm{Zi}$. For that reason we will use the same kind of notation which appears in that work.

3.1. Definition. Let $\alpha, \beta \in \mathbb{Z}^{d}, \alpha \leq \beta$ (coordinatewise) and $[\alpha ; \beta]:=\left\{\mathfrak{x} \in \mathbb{Z}^{d}\right.$ : $\alpha \leq \mathfrak{x} \leq \beta\}$. For a function $M: \mathbb{Z}^{d} \rightarrow \mathscr{B}(X, Y)$ we define the restriction of $M$ to $G \subset \mathbb{Z}^{\bar{d}}$ by

$$
M_{G}(\mathfrak{x}):=\left\{\begin{array}{cll}
M(\mathfrak{x}) & : & \mathfrak{x} \in G, \\
0 & : & \mathfrak{x} \notin G .
\end{array}\right.
$$

The difference operators $\Delta^{e_{j}}(j=1, \ldots, d)$ are defined for the unit vectors $e_{j}$ of $\mathbb{Z}^{d}$ as

$$
\left(\Delta^{e_{j}} M_{[\alpha ; \beta]}\right)(\mathfrak{x}):=\left\{\begin{array}{cl}
M_{[\alpha ; \beta]}(\mathfrak{x})-M_{[\alpha ; \beta]}\left(\mathfrak{x}-e_{j}\right) & : \mathfrak{x}_{j} \neq \alpha_{j} \\
0 & : \mathfrak{x}_{j}=\alpha_{j}
\end{array}\right.
$$

For arbitrary $\gamma=\left(\gamma_{1}, \ldots, \gamma_{d}\right)=\sum_{j=1}^{d} \gamma_{j} e_{j}$ with $\gamma_{j} \in\{0,1\}$ we set

$$
\Delta^{0} M_{[\alpha ; \beta]}:=M_{[\alpha ; \beta]}, \quad \Delta^{\gamma} M_{[\alpha ; \beta]}:=\Delta^{\gamma_{1} e_{1}} \circ \cdots \circ \Delta^{\gamma_{d} e_{d}} M_{[\alpha ; \beta]} .
$$

Next we generalize the defintion of a variation.

3.2. Definition. Let $M: \mathbb{Z}^{d} \rightarrow \mathscr{B}(X, Y)$ be an arbitrary function and $\mathcal{T} \subset \mathscr{B}(X, Y)$ a bounded collection. We define the $\mathcal{T}$-variation of $M$ in the interval $[\alpha ; \beta]$ by

$$
\underset{[\alpha ; \beta]}{\operatorname{var}_{\mathcal{T}}} M_{[\alpha ; \beta]}:=\sum_{\mathfrak{x} \in[\alpha ; \beta]}\left\|\left(\Delta^{\gamma_{\mathfrak{x}}} M_{[\alpha ; \beta]}\right)(\mathfrak{x})\right\|_{\mathcal{T}}
$$

where $\gamma_{\mathfrak{x}}=\left(\gamma_{\mathfrak{x}_{1}}, \ldots, \gamma_{\mathfrak{x}_{d}}\right)$ with

$$
\gamma_{\mathfrak{x}_{j}}:=\left\{\begin{array}{lll}
1 & : & \mathfrak{x}_{j} \neq \alpha_{j} \\
0 & : & \mathfrak{x}_{j}=\alpha_{j}
\end{array}\right.
$$

Of course if $\mathcal{T}$ is the unit ball of $\mathscr{B}(X, Y)$, we have the usual notation of bounded variation, which we simply denote by $\operatorname{var}_{[\alpha ; \beta]} M_{[\alpha ; \beta]}$ without the subscript $\mathcal{T}$.

The next result is a practical tool to estimate the $\mathcal{T}$-variation of a (discrete) function.

3.3. Lemma. Let $\alpha_{n}, \beta_{n} \in \mathbb{Z}^{d}, \alpha_{n} \leq \beta_{n}$ and $\left(\left[\alpha_{n} ; \beta_{n}\right]\right)_{n \in \mathbb{N}}$ be a (disjoint) decomposition of $\mathbb{Z}^{d}$. If $F: \mathbb{R}^{d} \rightarrow \mathscr{B}(X, Y)$ is a sufficiently smooth function and the collection

$$
\mathcal{T}:=\bigcup_{n \in \mathbb{N}}\left\{\left(\beta_{n}-\alpha_{n}\right)^{\gamma}\left(D^{\gamma} F\right)(x): x \in\left[\alpha_{n}, \beta_{n}\right], \gamma \leq(1, \ldots, 1)\right\}
$$

is bounded, then the restriction of $F$ to $\mathbb{Z}^{d}$ satisfies

$$
\sup _{n \in \mathbb{N}\left[\alpha_{n} ; \beta_{n}\right]} \operatorname{var}_{\mathcal{T}} F_{\left[\alpha_{n} ; \beta_{n}\right]} \leq 2^{d} .
$$

Proof. Let $n \in \mathbb{N}$ be arbitrary. If we rearrange the sum in Definition 3.1 we obtain

$$
\underset{\left[\alpha_{n} ; \beta_{n}\right]}{\operatorname{ar}_{\mathcal{T}}} F_{\left[\alpha_{n} ; \beta_{n}\right]}=\sum_{\gamma \leq(1, \ldots, 1)} \sum_{\left\{\mathfrak{x}: \gamma_{\mathfrak{x}}=\gamma\right\}}\left\|\left(\Delta^{\gamma} F_{\left[\alpha_{n} ; \beta_{n}\right]}\right)(\mathfrak{x})\right\|_{\mathcal{T}},
$$

where the sum over $\gamma$ has $2^{d}$ summands. 
1. CASE: $\gamma=(0, \ldots, 0)$

In this case only $\mathfrak{x}=\alpha$ has the property $\gamma_{\mathfrak{x}}=\gamma$ and therefore

$$
\sum_{\left\{\mathfrak{x}: \gamma_{\mathfrak{x}}=\gamma\right\}}\left\|\left(\Delta^{\gamma} F_{\left[\alpha_{n} ; \beta_{n}\right]}\right)(\mathfrak{x})\right\|_{\mathcal{T}}=\left\|F_{\left[\alpha_{n} ; \beta_{n}\right]}\left(\alpha_{n}\right)\right\|_{\mathcal{T}}=\left\|F\left(\alpha_{n}\right)\right\|_{\mathcal{T}} \leq 1 .
$$

2. CASE: $(0, \ldots, 0) \neq \gamma \leq(1, \ldots, 1)$

Take $\mathfrak{x} \in\left[\alpha_{n} ; \beta_{n}\right]$ with $\gamma_{\mathfrak{x}}=\gamma$. Let $q_{1}, \ldots, q_{r}$ be the coordinate directions for which $\gamma_{\mathfrak{x}_{q}} \neq 0$. Now by definition of the difference operator and the fundamental theorem of calculus we get

$$
\begin{aligned}
& \left(\Delta^{\gamma_{\mathfrak{x}}} F_{\left[\alpha_{n} ; \beta_{n}\right]}\right)(\mathfrak{x})=\int_{\left[\mathfrak{x}_{q_{1}}-1, \mathfrak{x}_{q_{1}}\right]} \ldots \int_{\left[\mathfrak{x}_{q_{r}}-1, \mathfrak{x}_{q_{r}}\right]}\left(D^{\gamma} F\right)(\xi) d \xi_{q_{r}} \ldots d \xi_{q_{1}} \\
& \quad=\frac{1}{\left(\beta_{n}-\alpha_{n}\right)^{\gamma}} \int_{\left[\mathfrak{x}_{q_{1}}-1, \mathfrak{x}_{q_{1}}\right]} \ldots \int_{\left[\mathfrak{x}_{q_{r}}-1, \mathfrak{x}_{q_{r}}\right]}\left(\beta_{n}-\alpha_{n}\right)^{\gamma}\left(D^{\gamma} F\right)(\xi) d \xi_{q_{r}} \ldots d \xi_{q_{1}} .
\end{aligned}
$$

This immediately implies $\left(\Delta^{\gamma} F_{\left[\alpha_{n} ; \beta_{n}\right]}\right)(\mathfrak{x}) \in \frac{1}{\left(\beta_{n}-\alpha_{n}\right)^{\gamma}} \cdot \operatorname{aco}(\mathcal{T})$ and thus

$$
\sum_{\left\{\mathfrak{x}: \gamma_{\mathfrak{x}}=\gamma\right\}}\left\|\left(\Delta^{\gamma} F_{\left[\beta_{n} ; \alpha_{n}\right]}\right)(\mathfrak{x})\right\|_{\mathcal{T}} \leq \sum_{\left\{\mathfrak{x}: \gamma_{\mathfrak{x}}=\gamma\right\}} \frac{1}{\left(\beta_{n}-\alpha_{n}\right)^{\gamma}}=1 .
$$

The last equality holds because there are exactly $\left(\beta_{n}-\alpha_{n}\right)^{\gamma}$ different $\mathfrak{x}$ in $\left[\alpha_{n} ; \beta_{n}\right]$ with $\gamma_{\mathfrak{x}}=\gamma$. Thus the first case, (3.3) and (3.2) yield to the desired result.

The proof of the following result, which extends Stečkin's multilplier theorem, illustrates how the notion of bounded variation allows us to write a multiplier function as a sum of characteristic functions (cf. (3.6) below).

Let $I_{n}:=\left[\alpha_{n} ; \beta_{n}\right]$ with $\alpha_{n}=(-n,-n, \ldots,-n), \beta_{n}=(n, n, \ldots, n)$ and

$$
I(\gamma, n):=\left\{\mathfrak{x} \in I_{n}: \mathfrak{x}_{i}=-n \text { if } \gamma_{i}=0\right\} .
$$

3.4. Theorem. Let $X$ be a UMD-space, $Y$ an arbitrary Banach space and $1<p<$ $\infty$. Assume that the function $M: \mathbb{Z}^{d} \rightarrow \mathscr{B}(X, Y)$ satisfies

$$
\sum_{\mathfrak{x} \in I(\gamma, n)}\left\|\left(\Delta^{\gamma} M_{I_{n}}\right)(\mathfrak{x})\right\| \leq C<\infty
$$

for all $\gamma \leq(1, \ldots, 1)$ and all $n \in \mathbb{N}$. Then $M \in \mathscr{M}_{p}\left(\mathbb{Z}^{d} ; X, Y\right)$.

Proof. Using the same rearrangement as in (3.2) and the assumption (3.4) we have that

$$
\operatorname{var}_{I_{n}} M_{I_{n}}=\sum_{\gamma \leq(1, \ldots, 1)} \sum_{\mathfrak{x} \in I(\gamma, n)}\left\|\left(\Delta^{\gamma} M_{I_{n}}\right)(\mathfrak{x})\right\| \leq 2^{d} C .
$$

The point of this notation of bounded variation is that $M$ can be written as

$$
M_{\left[\alpha_{n} ; \beta_{n}\right]}=\sum_{\mathfrak{x} \in\left[\alpha_{i, n} ; \beta_{i, n}\right]}\left(\left(\Delta^{\gamma_{\mathfrak{x}}} M_{\left[\alpha_{n} ; \beta_{n}\right]}\right)(\mathfrak{x})\right) \chi_{\left[\mathfrak{x} ; \beta_{n}\right]} .
$$

For scalar valued $M$ this was checked in [Zi], Lemma 1.3 (ii) and we apply this identity to $y^{*}\left(M_{\left[\alpha_{n} ; \beta_{n}\right]}(\mathfrak{x}) x\right)$ for all $x \in X, y^{*} \in Y^{*}$.

Now for an arbitrary $f: \mathbb{T}^{d} \rightarrow X$ with supp $\hat{f} \subset I_{n}$ we obtain from (3.6)

$$
\mathscr{K}_{M_{I_{n}}} f=\sum_{\mathfrak{x} \in I_{n}}\left(\left(\Delta^{\gamma_{\mathfrak{x}}} M_{I_{n}}\right)(\mathfrak{x})\right) \sim \mathscr{K}_{\chi_{\left[\mathfrak{x} ; \beta_{n}\right]}} f,
$$


where $\left(\left(\Delta^{\gamma_{\mathfrak{x}}} M_{I_{n}}\right)(\mathfrak{x})\right)^{\sim}$ is the operator which arises from $\left(\Delta^{\gamma_{\mathfrak{x}}} M_{I_{n}}\right)(\mathfrak{x})$ in the same way as it was done in Lemma 2.6. Since $X$ is a UMD-space we know by [Zi] that there exists a constant $D$ such that

$$
\left\|\mathscr{K}_{\chi_{[\alpha ; \beta]}} f\right\| \leq D\|f\|
$$

for all intervals $[\alpha ; \beta]$. Hence by (3.7) and (3.5)

$$
\begin{aligned}
\left.\left\|\mathscr{K}_{M} f\right\|=\left\|\mathscr{K}_{M_{I_{n}}} f\right\| \leq D\|f\| \sum_{\mathfrak{x} \in I_{n}} \|\left(\Delta^{\gamma_{\mathfrak{x}}} M_{I_{n}}\right)(\mathfrak{x})\right) \| & \\
& =D \operatorname{var}_{I_{n}} M_{I_{n}}\|f\| \leq 2^{d} C D\|f\| .
\end{aligned}
$$

Since the functions $f$ with compact support $\hat{f}$ are dense in $L^{p}\left(\mathbb{T}^{d} ; X\right)$, the claim follows.

3.5. Corollary. If $X$ and $Y$ are arbitrary Banach spaces, and $M: \mathbb{Z}^{d} \rightarrow \mathscr{B}(X, Y)$ satisfies (3.4), then $\left\{M(\mathfrak{x}): \mathfrak{x} \in \mathbb{Z}^{d}\right\}$ is $R$-bounded.

Proof. This follows from [We, Lemma 2.4, and the representations (3.6) and (3.7).

To obtain more refined multiplier theorems that generalize the Marcinkiewicz multiplier theorem, we assume that $M$ is not of bounded variation on all of $\mathbb{Z}^{d}$ but only uniformly on certain partitions of $\mathbb{Z}^{d}$. As one might guess from Corollary 3.5. the $R$-boundedness will then be needed. The partitions we will use are the following ones:

(a) The coarse decomposition. Set $\mathbf{D}_{0}:=\{0\} \subset \mathbb{Z}^{d}$ and for $n=d r+j, r \in$ $\mathbb{N}_{0}, j \in\{1, \ldots, d\}$

$$
\begin{aligned}
\mathbf{D}_{n}:=\left\{\mathfrak{x}=\left(\mathfrak{x}_{1}, \ldots, \mathfrak{x}_{d}\right) \in \mathbb{Z}^{d}:\left|\mathfrak{x}_{1}\right|, \ldots,\left|\mathfrak{x}_{j-1}\right|<2^{r+1},\right. & 2^{r} \leq\left|\mathfrak{x}_{j}\right|<2^{r+1}, \\
& \left.\left|\mathfrak{x}_{j+1}\right|, \ldots,\left|\mathfrak{x}_{d}\right|<2^{r}\right\} .
\end{aligned}
$$

(b) The fine decomposition. For $\nu=\left(\nu_{1}, \ldots, \nu_{d}\right) \in \mathbb{N}_{0}^{d}$ we define

$$
\mathbf{D}_{\nu}:=I_{\nu_{1}} \times \ldots \times I_{\nu_{d}}
$$

where $I_{0}=\{0\}$ and $I_{n}=\left\{k \in \mathbb{Z}: 2^{n-1} \leq|k|<2^{n}\right\}(n \in \mathbb{N})$.

Since $\mathbf{D}=\mathbf{D}_{n}\left(\right.$ resp. $\left.\mathbf{D}_{\nu}\right)$ are unions of $s=2$ (resp. $s=2^{d}$ ) intervals, we can moreover define the $\mathcal{T}$-variation of $M$ with respect to the decompositions by

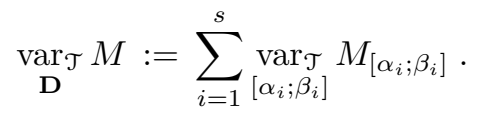

We are now able to define the generalized Marcinkiewicz conditions.

3.6. Definition. A function $M: \mathbb{Z}^{d} \rightarrow \mathscr{B}(X, Y)$ is said to be of bounded $\mathcal{T}_{\text {-variation }}$ with respect to the decompositions $\left(\mathbf{D}_{k}\right)$ (resp. $\left(\mathbf{D}_{\nu}\right)$ ), if there exist an $R$-bounded collection $\mathcal{T} \subset \mathscr{B}(X, Y)$ such that the condition $\left(\mathcal{M}_{c D}^{\mathcal{T}}\right)$

$$
\sup _{k \in \mathbb{N}_{0}} \operatorname{var}_{\mathbf{D}_{k}} M<\infty
$$

respectively $\left(\mathcal{M}_{f D}^{\mathcal{T}}\right)$

is fullfilled.

$$
\sup _{\nu \in \mathbb{N}_{0}^{d}} \operatorname{var}_{\mathbf{D}_{\nu}} M<\infty
$$


3.7. Theorem (operator-valued Marcinkiewicz theorem for $\left(\mathbf{D}_{k}\right)$ ). Let $X, Y$ be $U M D$-spaces and $1<p<\infty$. If the function $M: \mathbb{Z}^{d} \rightarrow \mathscr{B}(X, Y)$ possesses condition $\left(\mathcal{M}_{c D}^{\mathcal{T}}\right)$, then $M \in \mathscr{M}_{p}\left(\mathbb{Z}^{d} ; X, Y\right)$.

3.8. Theorem (operator-valued Marcinkiewicz theorem for $\left(\mathbf{D}_{\nu}\right)$ ). Let $X, Y$ be $U M D$-spaces with the property $(\alpha)$ and $1<p<\infty$. If the function $M: \mathbb{Z}^{d} \rightarrow$ $\mathscr{B}(X, Y)$ possesses condition $\left(\mathcal{M}_{f D}^{\mathcal{T}}\right)$, then $M \in \mathscr{M}_{p}\left(\mathbb{Z}^{d} ; X, Y\right)$.

3.9. Example. The Marcinkiewicz multiplier theorems in Bou2] and [Zi] are special cases of these theorems. In both papers one considers functions $M$ of the form $M(\mathfrak{x})=a(\mathfrak{x}) I, a(\mathfrak{x}) \in \mathbb{C}$. If we set $\mathcal{T}:=\left\{M(\mathfrak{x}): \mathfrak{x} \in \mathbb{Z}^{d}\right\}$, then using Remark 2.9 a) we get

$$
\begin{aligned}
& R(\mathcal{T}) \operatorname{var}_{\mathbf{D}} M=R(\mathcal{T}) \sum_{i} \operatorname{var}_{\left[\alpha_{i} ; \beta_{i}\right]} M_{\left[\alpha_{i} ; \beta_{i}\right]}=R(\mathcal{T}) \sum_{i} \sum_{\mathfrak{x} \in\left[\alpha_{i} ; \beta_{i}\right]}\left\|\left(\Delta^{\gamma_{\mathfrak{x}}} M_{\left[\alpha_{i} ; \beta_{i}\right]}\right)(\mathfrak{x})\right\|_{\mathcal{T}} \\
= & R(\mathcal{T}) \sum_{i} \sum_{\mathfrak{x} \in\left[\alpha_{i} ; \beta_{i}\right]}\left\|\left(\Delta^{\gamma_{\mathfrak{x}}} a_{\left[\alpha_{i} ; \beta_{i}\right]}\right)(\mathfrak{x}) I\right\|_{\mathcal{T}} \leq 2 \sum_{i} \sum_{\mathfrak{x} \in\left[\alpha_{i} ; \beta_{i}\right]}\left|\left(\Delta^{\gamma_{\mathfrak{x}}} a_{\left[\alpha_{i} ; \beta_{i}\right]}\right)(\mathfrak{x})\right|=2 \underset{\mathbf{D}}{\operatorname{var}} a .
\end{aligned}
$$

3.10. Remark. Since the elements $\mathbf{D}_{k}$ are unions of two intervals $\left[\alpha_{1 k} ; \beta_{1 k}\right],\left[\alpha_{2 k} ; \beta_{2 k}\right]$, the assumption of Theorem 3.7 implies in particular that the collection $\mathcal{S}=\left\{M\left(\alpha_{i k}\right)\right.$ : $\left.i=1,2 ; k \in \mathbb{N}_{0}\right\}$ has to be $R$-bounded (use Remark 2.9 b,(ii)).

Before we start to prove both theorems we list some further results, which will simplify the later arguments. For $G \subset \mathbb{Z}^{d}$ we define a characteristic multiplier function from $\mathbb{Z}^{d}$ to $\mathscr{B}(E)$ by

$$
\chi_{G}(\mathfrak{x}):=\left\{\begin{array}{lll}
I & : & \mathfrak{x} \in G \\
0 & : & \mathfrak{x} \notin G .
\end{array}\right.
$$

Now the following results hold

3.11. Theorem. Let $E$ be a UMD-space, $1<p<\infty$ and $S_{k}^{E}:=\mathscr{K}_{\chi_{\mathbf{D}_{k}}}, k \in \mathbb{N}_{0}$. Then there exist a $C_{p}>0$ such that for every trigonometric polynomial $f$

$$
\frac{1}{C_{p}}\|f\|_{L^{p}\left(\mathbb{T}^{d} ; E\right)} \leq\left\|\sum_{k=0}^{\infty} \varepsilon_{k} S_{k}^{E} f\right\|_{L^{p}\left(\Omega ; L^{p}\left(\mathbb{T}^{d} ; E\right)\right)} \leq C_{p}\|f\|_{L^{p}\left(\mathbb{T}^{d} ; E\right)} .
$$

A similar result is true for the fine decomposition. By $\left(\varepsilon_{\nu}\right)_{\nu \in \mathbb{N}_{0}^{d}}$ we denote an arbitrary $d$-dimensional renumeration of $\left(\varepsilon_{k}\right)_{k=0}^{\infty}$.

3.12. Theorem. Let $E$ be a UMD-space with the property $(\alpha), 1<p<\infty$ and $S_{\nu}^{E}:=\mathscr{K}_{\chi_{\mathbf{D}_{\nu}}}, \nu \in \mathbb{N}_{0}^{d}$. Then there exist a $C_{p}>0$ such that for every trigonometric polynomial $f$

$$
\frac{1}{C_{p}}\|f\|_{L^{p}\left(\mathbb{T}^{d} ; E\right)} \leq\left\|\sum_{\nu \in \mathbb{N}_{0}^{d}} \varepsilon_{\nu} S_{\nu}^{E} f\right\|_{L^{p}\left(\Omega ; L^{p}\left(\mathbb{T}^{d} ; E\right)\right)} \leq C_{p}\|f\|_{L^{p}\left(\mathbb{T}^{d} ; E\right)} .
$$

The proofs of both lemmas can be found in [Bou2] and [Zi]. Actually Zimmermann assumes for Theorem 3.12 that $E$ is a UMD-space with local unconditional structure. But his proof works also for our weaker assumption. The next result is a consequence of the Definition of a UMD-space and Lemma 2.5. The proof is implicitly in [Zi] (see also Lemma 7 in [Bou2] or Lemma 3.5 in [BG]). 
3.13. Lemma. Let $E$ be a UMD-space and $1<p<\infty$. Then the collection

$$
\mathcal{K}:=\left\{\mathscr{K}_{\chi_{G}}: G \text { is an interval in } \mathbb{Z}^{d}\right\} \subset \mathscr{B}\left(L^{p}\left(\mathbb{T}^{d} ; E\right)\right)
$$

is $R$-bounded.

3.14. Remark. The same statement also holds in the case $\mathbb{R}^{d}$.

Proof of Theorem 3.7. Let $\mathscr{K}_{n}:=\mathscr{K}_{M_{\mathbf{D}_{n}}}, n \in \mathbb{N}_{0}$. Now for any trigonometric polynomial we get

$$
S_{n}^{Y} \circ \mathscr{K}_{M} f=\mathscr{K}_{n} \circ S_{n}^{X} f
$$

and thus, using Theorem 3.11,

$$
\left\|\mathscr{K}_{M} f\right\|_{L^{p}\left(\mathbb{T}^{d} ; Y\right)} \leq C_{p}\left\|\sum_{n=0}^{\infty} \varepsilon_{n} \mathscr{K}_{n} \circ S_{n}^{X} f\right\|_{L^{p}\left(\Omega ; L^{p}\left(\mathbb{T}^{d} ; Y\right)\right)} .
$$

Now, if we can prove that the collection $\left\{\mathscr{K}_{n}: n \in \mathbb{N}_{0}\right\}$ is $R$-bounded, an additional application of Theorem 3.11 would complete this proof.

3.15. Lemma. The collection

$$
\left\{\mathscr{K}_{n}: n \in \mathbb{N}_{0}\right\} \subset \mathscr{B}\left(L^{p}\left(\mathbb{T}^{d} ; X\right), L^{p}\left(\mathbb{T}^{d} ; Y\right)\right)
$$

is R-bounded.

Proof of Lemma 3.15. By assumption there exist an $R$-bounded collection $\mathcal{T}$ with

$$
\underset{\mathbf{D}_{k}}{\operatorname{var}_{\mathcal{T}}} M=\sum_{i=1}^{2} \sum_{\mathfrak{x} \in\left[\alpha_{i, k} ; \beta_{i, k}\right]}\left\|\left(\Delta^{\gamma_{\mathfrak{x}}} M_{\left[\alpha_{i, k} ; \beta_{i, k}\right]}\right)(\mathfrak{x})\right\|_{\mathcal{T}} \leq C<\infty \quad \forall k \in \mathbb{N}_{0} .
$$

For the operator $\mathscr{K}_{n}$ we have the representation

$$
\mathscr{K}_{n}=\mathscr{K}_{M_{\left[\alpha_{1, n} ; \beta_{1, n}\right]}}+\mathscr{K}_{\left.M_{\left[\alpha_{2, n} ; \beta_{2, n}\right]}\right]} .
$$

Let us define the collection

$$
\mathcal{S}:=\operatorname{aco}(\tilde{\mathcal{T}}) \mathcal{K},
$$

where $\tilde{\mathcal{T}}$ is the collection from Lemma 2.6 and $\mathcal{K}$ is the one from Lemma 3.13. Using Lemma 2.4, Lemma 2.6 and Lemma 3.13 we get that $\mathcal{S}$ is $R$-bounded. Now using the representation formulas from (3.6) and (3.7) we obtain

$$
\mathscr{K}_{M_{\left[\alpha_{i, n} ; \beta_{i, n}\right]}}=\sum_{\mathfrak{x} \in\left[\alpha_{i, n} ; \beta_{i, n}\right]}\left(\left(\Delta^{\gamma_{\mathfrak{x}}} M_{\left[\alpha_{i, n} ; \beta_{i, n}\right]}\right)(\mathfrak{x})\right)^{\sim} \circ \mathscr{K}_{\chi_{\left[\mathfrak{x} ; \beta_{i, n}\right]}} .
$$

Since

$$
\begin{aligned}
& \left\|\left(\left(\Delta^{\gamma_{\mathfrak{x}}} M_{\left[\alpha_{i, n} ; \beta_{i, n}\right]}\right)(\mathfrak{x})\right)^{\sim} \circ \mathscr{K}_{\left.\chi_{\left[\mathfrak{x} ; \beta_{i, n}\right]}\right]}\right\|_{\mathcal{S}} \\
& \quad=\inf \left\{t>0:\left(\left(\Delta^{\gamma_{\mathfrak{x}}} M_{\left[\alpha_{i, n} ; \beta_{i, n}\right]}\right)(\mathfrak{x})\right)^{\sim} \circ \mathscr{K}_{\chi_{\left[\mathfrak{x} ; \beta_{i, n}\right]}} \in t \cdot \operatorname{aco}(\mathcal{S})\right\} \\
& \quad \leq \inf \left\{t>0:\left(\left(\Delta^{\gamma_{\mathfrak{x}}} M_{\left[\alpha_{i, n} ; \beta_{i, n}\right]}\right)(\mathfrak{x})\right)^{\sim} \circ \mathscr{K}_{\left[\mathfrak{x} ; \beta_{i, n}\right]} \in t \cdot \operatorname{aco}(\tilde{\mathcal{T}}) \mathcal{K}\right\} \\
& \quad \leq \inf \left\{t>0:\left(\left(\Delta^{\gamma_{\mathfrak{x}}} M_{\left[\alpha_{i, n} ; \beta_{i, n}\right]}\right)(\mathfrak{x})\right)^{\sim} \in t \cdot \operatorname{aco}(\tilde{\mathcal{T}})\right\} \\
& \quad=\inf \left\{t>0:\left(\Delta^{\gamma_{\mathfrak{x}}} M_{\left[\alpha_{i, n} ; \beta_{i, n}\right]}\right)(\mathfrak{x}) \in t \cdot \operatorname{aco}(\mathcal{T})\right\} \\
& \quad=\left\|\left(\Delta^{\gamma_{\mathfrak{x}}} M_{\left[\alpha_{i, n} ; \beta_{i, n}\right]}\right)(\mathfrak{x})\right\|_{\mathcal{T}}
\end{aligned}
$$

we thus obtain from (3.12) and Remark $2.9 \mathrm{~b}$,(i) that

$$
\left\|\mathscr{K}_{M_{\left[\alpha_{i, n} ; \beta_{i, n}\right]}}\right\|_{\mathcal{S}} \leq \sum_{\mathfrak{x} \in\left[\alpha_{i, n} ; \beta_{i, n}\right]}\left\|\left(\Delta^{\gamma_{\mathfrak{x}}} M_{\left[\alpha_{i, n} ; \beta_{i, n}\right]}\right)(\mathfrak{x})\right\|_{\mathcal{T}} .
$$


Taking (3.11), (3.10) and again Remark $2.9 \mathrm{~b}$,(i) this yields

$$
\left\|\mathscr{K}_{n}\right\|_{\mathcal{S}} \leq C<\infty \quad \forall n \in \mathbb{N}_{0}
$$

Applying Remark 2.9 b,(ii) the proof is complete.

3.16. Remark. The proofs of Theorem 3.7 and Lemma 3.15 showed that the operator norm of $\mathscr{K}_{M}$ can be estimated by

$$
\left\|\mathscr{K}_{M}\right\|_{\mathscr{B}\left(L^{p}\left(\mathbb{T}^{d} ; X\right), L^{p}\left(\mathbb{T}^{d} ; Y\right)\right)} \leq C R(\mathcal{T}) \sup _{k \in \mathbb{N}_{0}} \operatorname{var}_{\mathbf{D}_{k}} M
$$

where the constant $C$ only depends on $p$ and the dimension $d$, but not on the collection $\mathcal{T}$ and the multiplier $M$.

3.17. Remark. The proof of Theorem 3.8 works in the same way.

\section{The continuous CASE}

In the beginning of this section we'd like to present a criterion for the $R$ boundedness of an operator-valued function on $\mathbb{R}^{d}$. This will be done by using Corollary 3.5 of the preceding section, which already gave a tool on how to decide whether an operator-valued function on $\mathbb{Z}^{d}$ is $R$-bounded. Before stating the result, we need some additional notation.

Let $\xi \in \mathbb{R}^{d}, \gamma$ be a multiindex with $0 \neq \gamma \leq(1, \ldots, 1)$ and $q_{1}, \ldots, q_{r}$ be the coordinate directions for which $\gamma_{q_{i}}=1$. In this case we set $\xi_{\gamma}=\left(\xi_{q_{1}}, \ldots, \xi_{q_{r}}\right) \in \mathbb{R}^{r}$.

4.1. Theorem. Let $X, Y$ be arbitrary Banach spaces and $M: \mathbb{R}^{d} \rightarrow \mathscr{B}(X, Y)$ a bounded function with continuous derivatives $D^{\gamma} M, \gamma \leq(1, \ldots, 1)$. If moreover

$$
\int_{\mathbb{R}^{r}}\left\|\left(D^{\gamma} M\right)\left(\xi_{\gamma}\right)\right\| d \xi_{\gamma} \leq C<\infty
$$

for each $0 \neq \gamma \leq(1, \ldots, 1)$, then the collection

$$
\mathcal{M}:=\left\{M(x): x \in \mathbb{R}^{d}\right\}
$$

is R-bounded.

Proof. Set $M_{k}(x):=M\left(x / 2^{k}\right), k \in \mathbb{N}$, and restrict $M_{k}$ to $\mathbb{Z}^{d}$. Since $M$ is bounded, we know from Corollary 3.5 that the collection

$$
\mathcal{M}_{k}:=\left\{M_{k}(\mathfrak{x}): \mathfrak{x} \in \mathbb{Z}^{d}\right\}
$$

is $R$-bounded, if

$$
\sum_{\mathfrak{x} \in I(\gamma, n)}\left\|\left(\Delta^{\gamma} M_{k, I_{n}}\right)(\mathfrak{x})\right\| \leq \tilde{C}_{k}<\infty
$$

for each $0 \neq \gamma \leq(1, \ldots, 1)$ holds. Now, in analogy to the proof of Lemma 3.3, the fundamental theorem of calculus states that for $\mathfrak{x} \in I(\gamma, n)$ we have

$$
\left(\Delta^{\gamma} M_{k, I_{n}}\right)(\mathfrak{x})=\int_{\left[\mathfrak{x}_{q_{1}}-1, \mathfrak{x}_{q_{1}}\right]} \ldots \int_{\left[\mathfrak{x}_{q_{r}}-1, \mathfrak{x}_{q_{r}}\right]}\left(D^{\gamma} M_{k}\right)(\xi) d \xi_{q_{r}} \ldots d \xi_{q_{1}}
$$


and moreover

$$
\begin{aligned}
\sum_{\mathfrak{x} \in I(\gamma, n)}\left\|\left(\Delta^{\gamma} M_{k, I_{n}}\right)(\mathfrak{x})\right\| & \leq \int_{[-n, n]^{r}}\left\|\left(D^{\gamma} M_{k}\right)\left(\xi_{\gamma}\right)\right\| d \xi_{\gamma} \\
= & \int_{\left[-n / 2^{k}, n / 2^{k}\right]^{r}}\left\|\left(D^{\gamma} M\right)\left(\xi_{\gamma}\right)\right\| d \xi_{\gamma} \\
& \leq \int_{\mathbb{R}^{r}}\left\|\left(D^{\gamma} M\right)\left(\xi_{\gamma}\right)\right\| d \xi_{\gamma} \leq C .
\end{aligned}
$$

So by now we have proved that

$$
\sum_{\mathfrak{x} \in I(\gamma, n)}\left\|\left(\Delta^{\gamma} M_{k, I_{n}}\right)(\mathfrak{x})\right\| \leq C
$$

where the constant is independent of $k$. Since $\mathcal{M}_{k} \subset \mathcal{M}_{k+1}$, we obtain from Corollary 3.5 that the collection

$$
\bigcup_{k=1}^{\infty} \mathcal{M}_{k}=\left\{M\left(\mathfrak{x} / 2^{k}\right): \mathfrak{x} \in \mathbb{Z}^{d}, k \in \mathbb{N}\right\}
$$

is $R$-bounded. An application of Lemma 2.3 completes the proof.

The remaining part of this section is concerned with extensions of the Mikhlin multiplier theorems from Bou2, $\mathrm{McC}$ ] and [Zi] for scalar-valued multipliers to new theorems with operator-valued multiplier functions. In We the second author already considered the one-dimensional case and used it to give a new characterization of maximal $L^{p}$-regularity of abstract differential equations. In this paragraph we will generalize this result to the higher-dimensional setting using the Marcinkiewicz theorems from section 3 .

To be able to use the results of the preceeding section we apply the Poisson summation formula, as in section 2 of [Zi], and the following two lemmas.

4.2. Lemma. Let $E$ be a Banach space, $1 \leq p<\infty$ and $\varphi \in \mathscr{S}\left(\mathbb{R}^{d} ; E\right)$. Then we have

where

$$
\|\varphi\|_{L^{p}\left(\mathbb{R}^{d} ; E\right)}=\lim _{k \rightarrow \infty}\left\|\varphi_{k, p}\right\|_{L^{p}\left(\mathbb{T}^{d} ; E\right)}
$$

$$
\varphi_{k, p}(\mathfrak{x})=2^{-\frac{d k}{p^{\prime}}} \sum_{\mathfrak{x} \in \mathbb{Z}^{d}} \hat{\varphi}\left(\mathfrak{x} / 2^{k}\right) \otimes \mathfrak{e}_{\mathfrak{x}} \quad\left(1 / p+1 / p^{\prime}=1\right) .
$$

4.3. Lemma. Let $\left(M_{n}\right)_{n \in \mathbb{N}} \subset \mathscr{M}_{p}\left(\mathbb{R}^{d} ; X, Y\right)$ be a sequence of Fourier multipliers that converges almost everywhere to $M$. Then

$$
\left\|\mathscr{K}_{M}\right\|_{\mathscr{B}\left(L^{p}\left(\mathbb{T}^{d} ; X\right), L^{p}\left(\mathbb{T}^{d} ; Y\right)\right)} \leq \sup \left\{\left\|\mathscr{K}_{M_{n}}\right\|_{\mathscr{B}\left(L^{p}\left(\mathbb{T}^{d} ; X\right), L^{p}\left(\mathbb{T}^{d} ; Y\right)\right)}: n \in \mathbb{N}\right\} .
$$

We now state the first of two Mikhlin-type Fourier multiplier theorems

4.4. Theorem (First operator-valued Mikhlin theorem). Let $X, Y$ be UMD spaces and $1<p<\infty$. If the function $M: \mathbb{R}^{d} \backslash\{0\} \rightarrow \mathscr{B}(X, Y)$ has the property that their distributional derivatives $D^{\gamma} M$ of order $\gamma \leq(1, \ldots, 1)$ are represented by functions and moreover

$$
R\left(\left\{|x|^{|\gamma|}\left(D^{\gamma} M\right)(x): x \in \mathbb{R}^{d} \backslash\{0\}, \gamma \leq(1, \ldots, 1)\right\}\right)<\infty
$$

holds, then $M \in \mathscr{M}_{p}\left(\mathbb{R}^{d} ; X, Y\right)$. 
Proof. We will divide the proof into three steps.

Step 1: $M \in \mathscr{S}\left(\mathbb{R}^{d} ; \mathscr{B}(X, Y)\right)$

For $f \in \mathscr{S}\left(\mathbb{R}^{d} ; X\right)$ we now have

$$
\mathscr{K}_{M} f=(M(\cdot) \hat{f}(\cdot))^{\vee}=\check{M} * f \in \mathscr{S}\left(\mathbb{R}^{d} ; Y\right) .
$$

By applying Lemma 4.2 we thus obtain

$$
\begin{array}{r}
\left\|\mathscr{K}_{M} f\right\|_{L^{p}\left(\mathbb{R}^{d} ; Y\right)}=\lim _{k}\left\|\mathscr{K}_{M_{k}} f_{k}\right\|_{L^{p}\left(\mathbb{T}^{d} ; Y\right)} \\
\leq \sup \left\{\left\|\mathscr{K}_{M_{k}}\right\|_{\mathscr{B}\left(L^{p}\left(\mathbb{T}^{d} ; X\right), L^{p}\left(\mathbb{T}^{d} ; Y\right)\right)}: k \in \mathbb{N}\right\}\|f\|_{L^{p}\left(\mathbb{R}^{d} ; X\right)},
\end{array}
$$

where

$$
M_{k}(\mathfrak{x}):=M\left(\mathfrak{x} / 2^{k}\right), \quad f_{k}(\mathfrak{x})=2^{-\frac{d k}{p^{\prime}}} \sum_{\mathfrak{x} \in \mathbb{Z}^{d}} \hat{f}\left(\mathfrak{x} / 2^{k}\right) \otimes \mathfrak{e}_{\mathfrak{x}}, \quad \mathfrak{x} \in \mathbb{Z}^{d} .
$$

The goal of the next calculation is to show that

$$
\left\|\mathscr{K}_{M_{k}}\right\|_{\mathscr{B}\left(L^{p}\left(\mathbb{T}^{d} ; X\right), L^{p}\left(\mathbb{T}^{d} ; Y\right)\right)} \leq C \quad \forall k \in \mathbb{N} .
$$

This will be done by using Theorem 3.7. For that reason we have to secure that the Marcinkiewicz conditions $\left(\mathcal{M}_{c D}^{\mathcal{T}_{k}}\right)$ hold for each $M_{k}$ with Marcinkiewicz constants that can be estimated independently of $k$ (see Remark 3.16). For the $R$-bounded collection $\mathcal{T}_{k}$ we choose

$\mathcal{T}_{k}:=\bigcup_{i \in\{1,2\}, n \in \mathbb{N}_{0}}\left\{\left(\beta_{i, n}-\alpha_{i, n}\right)^{\gamma}\left(D^{\gamma} M\left(\cdot / 2^{k}\right)\right)(x): x \in\left[\alpha_{i, n}, \beta_{i, n}\right], \gamma \leq(1, \ldots, 1)\right\}$.

Here $\left(\left[\alpha_{i, n} ; \beta_{i, n}\right]\right)_{i, n}$ is the coarse decomposition. By definition of the $\mathcal{T}$-variation we have $\left(n \in \mathbb{N}_{0}\right)$

$$
\operatorname{var}_{\mathcal{D}_{n}} M_{k}=\sum_{i=1}^{2} \sum_{\mathfrak{x} \in\left[\alpha_{i, n} ; \beta_{i, n}\right]}\left\|\left(\Delta^{\gamma_{\mathfrak{x}}} M_{\left[\alpha_{i, n} ; \beta_{i, n}\right]}\left(\cdot / 2^{k}\right)\right)(\mathfrak{x})\right\|_{\mathcal{T}_{k}} .
$$

Using Lemma 3.3, we obtain

$$
\sup _{n \in \mathbb{N}_{0}} \operatorname{var}_{\mathcal{T}_{k}} M_{k} \leq 2^{d}
$$

To apply Theorem 3.7 and Remark 3.16 we have to estimate $R\left(\mathcal{T}_{k}\right)$. If we define $\Delta_{0}=\{0\} \subset \mathbb{R}^{d}$ and for $n=d r+j, r \in \mathbb{N}_{0}, j \in\{1, \ldots, d\}$

$$
\begin{aligned}
\Delta_{n}:=\left\{x=\left(x_{1}, \ldots, x_{d}\right) \in \mathbb{R}^{d}:\left|x_{1}\right|, \ldots,\left|x_{j-1}\right|<2^{r+1},\right. & 2^{r} \leq\left|x_{j}\right|<2^{r+1}, \\
& \left.\left|x_{j+1}\right|, \ldots,\left|x_{d}\right|<2^{r}\right\},
\end{aligned}
$$

then from the definition of the coarse decomposition we know that (note that the following constant $r$ depends on $n$ )

- The sizes of the edges of the two subcubes $\left[\alpha_{1, n} ; \beta_{1, n}\right],\left[\alpha_{2, n} ; \beta_{2, n}\right]$ of $\mathbf{D}_{n}$ are not greater than $2^{r+1}$,

- $|x|_{\infty} \geq 2^{r}$ for all $x \in \Delta_{n}(n \geq 1)$.

Now let $x \in\left[\alpha_{i, n} ; \beta_{i, n}\right]$ be arbitrary. Thus

$$
\left(\beta_{i, n}-\alpha_{i, n}\right)^{\gamma}\left(D^{\gamma} M\left(\cdot / 2^{k}\right)\right)(x)=2^{|\gamma|} \frac{\left(\beta_{i, n}-\alpha_{i, n}\right)^{\gamma}}{2^{(r+1)|\gamma|}} 2^{(r-k)|\gamma|}\left(D^{\gamma} M\right)\left(x / 2^{k}\right)
$$


and therefore (use Remark 2.2 (d))

$$
\begin{aligned}
R & \left(\mathcal{T}_{k}\right) \leq C_{1} R\left(\bigcup_{i, n}\left\{2^{(r-k)|\gamma|}\left(D^{\gamma} M\right)\left(x / 2^{k}\right): x \in\left[\alpha_{i, n}, \beta_{i, n}\right], \gamma \leq(1, \ldots, 1)\right\}\right) \\
& =C_{1} R\left(\bigcup_{n}\left\{2^{(r-k)|\gamma|}\left(D^{\gamma} M\right)\left(x / 2^{k}\right): x \in \Delta_{n}, \gamma \leq(1, \ldots, 1)\right\}\right) \\
& =C_{1} R\left(\bigcup_{n}\left\{\frac{2^{(r-k)|\gamma|}}{|x|^{|\gamma|}} \cdot|x|^{|\gamma|}\left(D^{\gamma} M\right)(x): x \in \frac{1}{2^{k}} \Delta_{n}, \gamma \leq(1, \ldots, 1)\right\}\right) \\
& \leq C_{2} R\left(\bigcup_{n}\left\{|x|^{|\gamma|}\left(D^{\gamma} M\right)(x): x \in \frac{1}{2^{k}} \Delta_{n}, \gamma \leq(1, \ldots, 1)\right\}\right) \\
& \leq C_{2} R\left(\left\{|x|^{|\gamma|}\left(D^{\gamma} M\right)(x): x \in \mathbb{R}^{d} \backslash\{0\}, \gamma \leq(1, \ldots, 1)\right\}\right) .
\end{aligned}
$$

So (4.3), (4.2) and Remark 3.16 yield

$$
\left\|\mathscr{K}_{M}\right\| \leq C R\left(\left\{|x|^{|\gamma|}\left(D^{\gamma} M\right)(x): x \in \mathbb{R}^{d} \backslash\{0\}, \gamma \leq(1, \ldots, 1)\right\}\right),
$$

where $C$ does not depend on the multiplier function $M$.

Step 2: $M$ is infinitely often differentiable.

Fix an infinitely often differentiable (scalar) function $\varrho$ with compact support such that $\varrho(0)=1$. Define for all $\varepsilon>0, \varrho_{\varepsilon}(\cdot):=\varrho(\varepsilon \cdot)$. Now $M_{\varepsilon}:=\varrho_{\varepsilon} M \in$ $\mathscr{S}\left(\mathbb{R}^{d} ; \mathscr{B}(X, Y)\right)$ converges pointwise to $M$ as $\varepsilon$ goes to 0 . By Lemma 4.3 and (4.4) we get

$$
\begin{aligned}
\left\|\mathscr{K}_{M}\right\| \leq & \sup \left\{\left\|\mathscr{K}_{M_{\varepsilon}}\right\|: \varepsilon>0\right\} \\
& \leq C \sup _{\varepsilon} R\left(\left\{|x|^{|\gamma|}\left(D^{\gamma} M_{\varepsilon}\right)(x): x \in \mathbb{R}^{d} \backslash\{0\}, \gamma \leq(1, \ldots, 1)\right\}\right) .
\end{aligned}
$$

By Leibniz's formula we have

$$
|x|^{|\gamma|}\left(D^{\gamma} M_{\varepsilon}\right)(x)=\sum_{\alpha+\beta=\gamma} C_{\alpha, \beta}|x|^{|\alpha|}\left(D^{\alpha} M\right)(x)|x|^{|\beta|}\left(D^{\beta} \varrho_{\varepsilon}\right)(x) .
$$

The $R$-bound of each term in the sum can be estimated by

$$
\begin{aligned}
R\left(\left\{|x|^{|\alpha|}\left(D^{\alpha} M\right)(x)|x|^{|\beta|}\left(D^{\beta} \varrho_{\varepsilon}\right)(x): x\right.\right. & \left.\left.\in \mathbb{R}^{d} \backslash\{0\}\right\}\right) \\
& \leq R\left(\left\{|x|^{|\alpha|}\left(D^{\alpha} M\right)(x): x \neq 0\right\}\right) \cdot \sup _{\varepsilon}\left\{|x|^{|\beta|}\left(D^{\beta} \varrho_{\varepsilon}\right)(x): x \neq 0\right\},
\end{aligned}
$$

where the supremum is independent of $\varepsilon$. Therefore Remark 2.2 (d) and (4.5) yield

$$
\begin{aligned}
& \left\|\mathscr{K}_{M}\right\| \leq C \sup _{\varepsilon} R\left(\left\{|x|^{|\gamma|}\left(D^{\gamma} M_{\varepsilon}\right)(x): x \in \mathbb{R}^{d} \backslash\{0\}, \gamma \leq(1, \ldots, 1)\right\}\right) \\
& \leq C_{1} \sup _{\varepsilon, \gamma} \sum_{\alpha+\beta=\gamma} C_{\alpha, \beta} R\left(\left\{|x|^{|\alpha|}\left(D^{\alpha} M\right)(x)|x|^{|\beta|}\left(D^{\beta} \varrho_{\varepsilon}\right)(x): x \in \mathbb{R}^{d} \backslash\{0\}\right\}\right) \\
& \quad \leq C_{2} R\left(\left\{|x|^{|\gamma|}\left(D^{\gamma} M\right)(x): x \in \mathbb{R}^{d} \backslash\{0\}, \gamma \leq(1, \ldots, 1)\right\}\right) .
\end{aligned}
$$

Here $C_{2}$ is again independent of $M$.

Step 3: $M$ arbitrary as in the assumption.

Choose an infinitely often differentiable (scalar) function $\varrho$ with $\varrho \geq 0,\|\varrho\|_{1}=1$ and $\operatorname{supp} \varrho \subset[-1,1]^{d}$. For $\varepsilon>0$ define $\varrho_{\varepsilon}(\cdot):=\varepsilon^{-d} \varrho(\cdot / \varepsilon)$. Now $M_{\varepsilon}:=M * \varrho_{\varepsilon}$ is 
infinitely often differentiable and converges almost everywhere to $M$ as $\varepsilon$ converges to 0 . The result from the second step in connection with Lemma 4.3 lead to

$$
\begin{aligned}
\left\|\mathscr{K}_{M}\right\| & \leq \sup \left\{\left\|\mathscr{K}_{M_{\varepsilon}}\right\|: \varepsilon>0\right\} \\
& \leq C \sup _{\varepsilon} R\left(\left\{|x|^{|\gamma|}\left(D^{\gamma}\left[M * \varrho_{\varepsilon}\right]\right)(x): x \in \mathbb{R}^{d} \backslash\{0\}, \gamma \leq(1, \ldots, 1)\right\}\right) .
\end{aligned}
$$

Since for arbitrary $\gamma \leq(1, \ldots, 1)$

$$
\begin{aligned}
& R\left(\left\{|x|^{|\gamma|}\left(D^{\gamma}\left[M * \varrho_{\varepsilon}\right]\right)(x): 0<|x|_{\infty} \leq 2 \varepsilon\right\}\right) \\
& \quad=R\left(\left\{\frac{|x|^{|\gamma|}}{(2 \varepsilon)^{|\gamma|}} \cdot(2 \varepsilon)^{|\gamma|} \cdot\left\|D^{\gamma} \varrho_{\varepsilon}\right\|_{1} \cdot\left(M * \frac{D^{\gamma} \varrho_{\varepsilon}}{\left\|D^{\gamma} \varrho_{\varepsilon}\right\|_{1}}\right)(x): 0<|x|_{\infty} \leq 2 \varepsilon\right\}\right) \\
& \quad \leq C_{1}(2 \varepsilon)^{|\gamma|} \cdot\left\|D^{\gamma} \varrho_{\varepsilon}\right\|_{1} \cdot R\left(\left\{M(x): x \in \mathbb{R}^{d} \backslash\{0\}\right\}\right) \\
& \quad=C_{1} 2^{|\gamma|} \cdot\left\|D^{\gamma} \varrho\right\|_{1} \cdot R\left(\left\{M(x): x \in \mathbb{R}^{d} \backslash\{0\}\right\}\right),
\end{aligned}
$$

we thus in particular obtain

$$
\begin{aligned}
R\left(\left\{| x | ^ { | \gamma | } \left(D^{\gamma}[M\right.\right.\right. & \left.\left.\left.\left.* \varrho_{\varepsilon}\right]\right)(x): 0<|x|_{\infty} \leq 2 \varepsilon, \gamma \leq(1, \ldots, 1)\right\}\right) \\
& \leq C_{2} R\left(\left\{|x|^{|\gamma|}\left(D^{\gamma} M\right)(x): x \in \mathbb{R}^{d} \backslash\{0\}, \gamma \leq(1, \ldots, 1)\right\}\right) .
\end{aligned}
$$

The above estimations are consequences of Remark 2.2 (d) and Lemma 2.3.

For arbitrary $\varepsilon>0, \varrho_{\varepsilon}$ has its support in $[-\varepsilon, \varepsilon]^{d}$ and so for any $\alpha \leq(1, \ldots, 1)$ and each $|x|_{\infty} \geq 2 \varepsilon$

$$
\begin{aligned}
& |x|^{|\alpha|}\left(D^{\alpha}\left[M * \varrho_{\varepsilon}\right]\right)(x)=|x|^{|\alpha|} \int_{\{\xi:|x-\xi| \leq \varepsilon\}}\left(D^{\alpha} M\right)(\xi) \varrho_{\varepsilon}(x-\xi) d \xi \\
& \quad=\int_{\{\xi:|x-\xi| \leq \varepsilon\}}|\xi|^{|\alpha|}\left(D^{\alpha} M\right)(\xi) \cdot \frac{|x|^{|\alpha|}}{|\xi|^{|\alpha|}} \varrho_{\varepsilon}(x-\xi) d \xi \\
& \quad=C_{1} \int_{\{\xi:|x-\xi| \leq \varepsilon\}}|\xi|^{|\alpha|}\left(D^{\alpha} M\right)(\xi) \cdot \frac{|x|^{|\alpha|}}{C_{1}|\xi|^{|\alpha|}} \varrho_{\varepsilon}(x-\xi) d \xi,
\end{aligned}
$$

where $C_{1}$ is a constant which fulfills for all $\alpha$ and $\varepsilon>0$

$$
\sup \left\{\frac{|x|^{|\alpha|}}{|\xi|^{|\alpha|}}:|x-\xi| \leq \varepsilon,|x|_{\infty} \geq 2 \varepsilon\right\} \leq C_{1} .
$$

Since $\left\|\varrho_{\varepsilon}\right\|_{1}=1$ we obtain that for each $x$ with $|x|_{\infty} \geq 2 \varepsilon$

$$
\begin{aligned}
\int_{\{\xi:|x-\xi| \leq \varepsilon\}}|\xi|^{|\alpha|}\left(D^{\alpha} M\right)(\xi) \cdot \frac{|x|^{|\alpha|}}{C_{1}|\xi|^{|\alpha|}} \varrho_{\varepsilon}(x-\xi) d \xi \\
\in \operatorname{aco}\left(\left\{|x|^{|\alpha|}\left(D^{\alpha} M\right)(x): x \in \mathbb{R}^{d} \backslash\{0\}\right\}\right)
\end{aligned}
$$

and thus by Lemma 2.3

$$
\begin{aligned}
R\left(\left\{| x | ^ { | \gamma | } \left(D^{\gamma} M\right.\right.\right. & \left.\left.\left.* \varrho_{\varepsilon}\right)(x):|x|_{\infty} \geq 2 \varepsilon, \gamma \leq(1, \ldots, 1)\right\}\right) \\
& \leq C_{2} R\left(\left\{|x|^{|\gamma|}\left(D^{\gamma} M\right)(x): x \in \mathbb{R}^{d} \backslash\{0\}, \gamma \leq(1, \ldots, 1)\right\}\right) .
\end{aligned}
$$

Using (4.6), (4.7) the theorem is proved.

4.5. Theorem (Second operator-valued Mikhlin theorem). If $X$ and $Y$ are UMDspaces with the property $(\alpha)$ and where the function $M: \mathbb{R}^{d} \backslash\{0\} \rightarrow \mathscr{B}(X, Y)$ has 
the property that their distributional derivatives $D^{\gamma} M$ of order $\gamma \leq(1, \ldots, 1)$ are represented by functions which fulfill

$$
R\left(\left\{x^{\gamma}\left(D^{\gamma} M\right)(x): x \in \mathbb{R}^{d} \backslash\{0\}, \gamma \leq(1, \ldots, 1)\right\}\right)<\infty,
$$

then $M \in \mathscr{M}_{p}\left(\mathbb{R}^{d} ; X, Y\right)(1<p<\infty)$.

Proof. Again we divide the proof into three steps.

Step 1: $M \in \mathscr{S}\left(\mathbb{R}^{d} ; \mathscr{B}(X, Y)\right)$

Arguing as in the first step of the proof of Theorem 4.4 we obtain in analogy to (4.3)

$$
\sup _{\nu \in \mathbb{N}_{0}^{d}} \operatorname{var}_{\mathcal{T}_{\mathrm{k}}} M_{k} \leq 2^{d},
$$

where the collection $\mathcal{T}_{k}$ is defined by

$$
\mathcal{T}_{k}:=\bigcup_{\substack{i \in\left\{1, \ldots, 2^{d}\right\} \\ \nu \in \mathbb{N}_{0}^{d}}}\left\{\left(\beta_{i, \nu}-\alpha_{i, \nu}\right)^{\gamma}\left(D^{\gamma} M\left(\cdot / 2^{k}\right)\right)(x): x \in\left[\alpha_{i, \nu}, \beta_{i, \nu}\right], \gamma \leq(1, \ldots, 1)\right\} .
$$

Here $\left(\left[\alpha_{i, \nu} ; \beta_{i, \nu}\right]\right)_{i, \nu}$ is the fine decomposition. Again it remains to show that $\mathcal{T}_{k}$ is $R$-bounded. Now we define for $\nu=\left(\nu_{1}, \ldots, \nu_{d}\right) \in \mathbb{Z}^{d}$

$$
\Delta_{\nu}:=\left\{x \in \mathbb{R}^{d} \backslash\{0\}: 2^{\nu_{i}-1} \leq\left|x_{i}\right|<2^{\nu_{i}} \text { for } i \in\{1, \ldots, d\}\right\} .
$$

For the fine decomposition we know

- The sizes of the edges of the $2^{d}$ subcuboids $\left[\alpha_{i, \nu} ; \beta_{1, \nu}\right]$ of $\mathbf{D}_{\nu}$ in the $j$-th coordinate direction are not larger than $2^{\nu_{j}}$.

- For $x \in \Delta_{\nu},\left(\nu \in \mathbb{N}^{d}\right)$ we have $\left|x_{j}\right| \geq 2^{\nu_{j}-1}$.

If $x \in\left[\alpha_{i, \nu} ; \beta_{i, \nu}\right]$ is arbitrary, we use the identity $\left(\left(2^{\alpha}\right):=\left(2^{\alpha_{1}}, \ldots, 2^{\alpha_{d}}\right), \alpha \in \mathbb{Z}^{d}\right)$

$$
\left(\beta_{i, \nu}-\alpha_{i, \nu}\right)^{\gamma}\left(D^{\gamma} M\left(\cdot / 2^{k}\right)\right)(x)=\frac{\left(\beta_{i, n}-\alpha_{i, n}\right)^{\gamma}}{\left(2^{\nu}\right)^{\gamma}} \cdot\left(2^{\nu}\right)^{\gamma} \cdot 2^{-k|\gamma|}\left(D^{\gamma} M\right)\left(x / 2^{k}\right)
$$

and Remark 2.2 (d) to get

$$
\begin{aligned}
& R\left(\mathcal{T}_{k}\right) \leq C_{1} R\left(\bigcup_{i, \nu}\left\{\frac{\left(2^{\nu}\right)^{\gamma}}{2^{k|\gamma|}}\left(D^{\gamma} M\right)\left(x / 2^{k}\right): x \in\left[\alpha_{i, \nu}, \beta_{i, \nu}\right], \gamma \leq(1, \ldots, 1)\right\}\right) \\
& =C_{1} R\left(\bigcup_{\nu}\left\{\left(2^{\nu}\right)^{\gamma} \cdot 2^{-k|\gamma|}\left(D^{\gamma} M\right)\left(x / 2^{k}\right): x \in \Delta_{\nu}, \gamma \leq(1, \ldots, 1)\right\}\right) \\
& =C_{1} R\left(\bigcup_{\nu}\left\{\frac{\left(2^{\nu}\right)^{\gamma} \cdot 2^{-k|\gamma|}}{x^{\gamma}} \cdot x^{\gamma}\left(D^{\gamma} M\right)(x): x \in \frac{1}{2^{k}} \Delta_{\nu}, \gamma \leq(1, \ldots, 1)\right\}\right) \\
& \leq C_{2} R\left(\bigcup_{\nu}\left\{x^{\gamma}\left(D^{\gamma} M\right)(x): x \in \frac{1}{2^{k}} \Delta_{\nu}, \gamma \leq(1, \ldots, 1)\right\}\right) \\
& \leq C_{2} R\left(\left\{x^{\gamma}\left(D^{\gamma} M\right)(x): x \in \mathbb{R}^{d} \backslash\{0\}, \gamma \leq(1, \ldots, 1)\right\}\right)
\end{aligned}
$$

and therefore

$$
\left\|\mathscr{K}_{M}\right\| \leq C R\left(\left\{x^{\gamma}\left(D^{\gamma} M\right)(x): x \in \mathbb{R}^{d} \backslash\{0\}, \gamma \leq(1, \ldots, 1)\right\}\right),
$$

where $C$ does not depend on the multiplier function $M$.

Step 2: The case where $M$ is infinitely often differentiable can be treated in the same way as in Theorem 4.4.

Step 3: $M$ fulfills the assumption of Theorem 4.5. 
For this step we modify the first part of the proof of Theorem 4.4 and obtain similarly to (4.7) for all $0<\varepsilon \leq 1$

$$
\begin{aligned}
R\left(\left\{x^{\gamma}\left(D^{\gamma}\left[M * \varrho_{\varepsilon}\right]\right)\right.\right. & \left.\left.(x): 0<|x|_{\infty} \leq \varepsilon, \gamma \leq(1, \ldots, 1)\right\}\right) \\
& \leq C R\left(\left\{x^{\gamma}\left(D^{\gamma} M\right)(x): x \in \mathbb{R}^{d} \backslash\{0\}, \gamma \leq(1, \ldots, 1)\right\}\right) .
\end{aligned}
$$

The remaining part will be treated in the following way:

Choose $\gamma \leq(1, \ldots, 1)$ arbitrary. Applying the binomial formula we get

$$
x^{\gamma}\left(D^{\gamma}\left[M * \varrho_{\varepsilon}\right]\right)(x)=\sum_{\alpha+\beta \leq \gamma} C_{\alpha, \beta}\left(\left(\xi^{\alpha}\left(D^{\alpha} M\right)(\xi)\right) *\left(\xi^{\beta}\left(D^{\beta} \varrho_{\varepsilon}\right)(\xi)\right)\right)(x) .
$$

Since supp $\varrho_{\varepsilon} \subset[-\varepsilon, \varepsilon]^{d}$, our assumption on the function $M$ enables us to write the above convolution for each $|x|_{\infty}>\varepsilon$ as follows:

$$
x^{\gamma}\left(D^{\gamma}\left[M * \varrho_{\varepsilon}\right]\right)(x)=\sum_{\alpha+\beta=\gamma} C_{\alpha, \beta} \int_{[-\varepsilon, \varepsilon]^{d}}(x-\xi)^{\alpha}\left(D^{\alpha} M\right)(x-\xi) \xi^{\beta}\left(D^{\beta} \varrho_{\varepsilon}\right)(\xi) d \xi .
$$

Now a similar argument as used in step 3 of the proof of Theorem 4.3 yields

$$
\begin{aligned}
R\left(\left\{x^{\gamma}\left(D^{\gamma}\left[M * \varrho_{\varepsilon}\right]\right)(x):|x|_{\infty}>\varepsilon\right\}\right) \\
\leq C_{1} R\left(\left\{x^{\alpha}\left(D^{\alpha} M\right)(x): x \in \mathbb{R}^{d} \backslash\{0\}, \alpha \leq(1, \ldots, 1)\right\}\right) \\
\cdot \sup _{\varepsilon}\left\{\left\|\xi^{\beta}\left(D^{\beta} \varrho_{\varepsilon}\right)(\xi)\right\|: \beta \leq(1, \ldots, 1)\right\} \\
=C_{1} R\left(\left\{x^{\alpha}\left(D^{\alpha} M\right)(x): x \in \mathbb{R}^{d} \backslash\{0\}, \alpha \leq(1, \ldots, 1)\right\}\right) \\
\cdot \sup \left\{\left\|\xi^{\beta}\left(D^{\beta} \varrho\right)(\xi)\right\|: \beta \leq(1, \ldots, 1)\right\}
\end{aligned}
$$

and thus also

$$
\begin{aligned}
R\left(\left\{x^{\gamma}\left(D^{\gamma}\left[M * \varrho_{\varepsilon}\right]\right)(x)\right.\right. & \left.\left.:|x|_{\infty}>\varepsilon, \gamma \leq(1, \ldots, 1)\right\}\right) \\
& \leq C_{2} R\left(\left\{x^{\gamma}\left(D^{\gamma} M\right)(x): x \in \mathbb{R}^{d} \backslash\{0\}, \gamma \leq(1, \ldots, 1)\right\}\right) .
\end{aligned}
$$

Together with (4.9) this completes the proof.

Note. Observe that the weight function $|x|^{|\gamma|}$ of Theorem 4.4 is larger than $\left|x^{\gamma}\right|$ from Theorem 4.5.

4.6. Remark. i) Theorem 4.4 and Theorem 4.5 are generalizations of Proposition 3 in $\mathrm{Zi}$.

ii) If $X$ and $Y$ are Hilbert spaces, then the unit ball of $\mathscr{B}(X, Y)$ is $R$-bounded and both theorems reduce to the result of Schwartz, which assumes that

$$
\left\|x^{\gamma}\left(D^{\gamma} M\right)(x)\right\| \leq C<\infty
$$

for all $x \in \mathbb{R}^{d} \backslash\{0\}$ and $\gamma \leq(1, \ldots, 1)$.

\section{REFERENCES}

[BG] E. Berkson and T.A. Gillespie, Spectral decompositions and harmonic analysis on UMD spaces, Studia Math. 112, (1994), no. 1, 13-49. MR1307598 (96c:42022)

[BL] J. Bergh and J. Löfström, Interpolation Spaces, Springer 1976. MR0482275 (58:2349)

[Bl] S. Blunck, Maximal regularity of discrete and continuous time evolution equations, Studia Math. 146 (2001), 157-176. MR1853519 (2002g:34125)

[Bou1] J. Bourgain, Some remarks on Banach spaces in which martingale difference sequences are unconditional, Arkiv Math. 21 (1983), 163-168. MR0727340 (85a:46011)

[Bou2] Vector-valued Singular Integrals and the $H^{1}-B M O$ Duality, Probability Theory and Harmonic Analysis, Monographs and Textbooks in Pure and Appl. Math. 98, Marcel Dekker, New York, 1986, 1-19. MR0830227 (87j:42049b) 
[Bu] D. Burkholder, A geometric condition that implies the existence of certain singular integrals of Banach-space-valued functions, Proc. of Conf. on Harmonic Analysis in Honor of Antoni Zygmund, Chicago 1981, Wadsworth Publishers, Belmont, Calif., 1983, 270-286. MR0730072 (85i:42020)

[CPSW] Ph. Clément, B. de Pagter, F.A. Sukochev and H. Witvliet, Schauder Decompositions and Multiplier Theorems, Studia Mathematica 138 (2000), 135-163. MR1749077 (2002c:47036)

[DJT] J. Diestel, H. Jarchow and A. Tonge, Absolutely summing operators, Cambridge University Press, 1995. MR.1342297 (96i:46001)

[LT] J. Lindenstrauss and L. Tzafriri, Classical Banach Spaces II (Function Spaces), SpringerVerlag 1979. MR0540367 (81c:46001)

[McC] T. Mc Connell, On Fourier multiplier transformations of Banach-valued functions, Trans. Amer. Math. Soc. 285 (1984), 739-757. MR0752501(87a:42033)

[Pi1] G. Pisier, Les inegalités de Khintchine-Kahane d'après C. Borel, Seminaire sur la ǵeométrie des espaces de Banach 7, (1977-1978), Ecole Polytechnique, Paris. MR0520209 (81c:60005)

[Pi2] Some results on Banach spaces without local unconditional structure, Comp. Math. 37 (1978), 3-19. MR0501916 (80e:46012)

[We] L. Weis, Operator-Valued Fourier Multiplier Theorems and Maximal $L^{p}$-regularity, Math. Ann. 319 (2001), 735-758. MR1825406(2002c:42016)

[Zi] F. Zimmermann, On vector-valued Fourier multiplier theorems, Studia Mathematica, T. XCIII (1989), 201-222. MR1030488 (91b:46031)

Institute of Mathematics I, University of Karlsruhe, Englerstrasse 2, D-76128 KarLSRUhe, Germany

E-mail address: zeljko.strkalj@math.uni-karlsruhe.de

Institute of Mathematics I, University of Karlsruhe, Englerstrasse 2, D-76128 KarLSRUhe, Germany

E-mail address: lutz.weis@math.uni-karlsruhe.de 